\title{
Bibliographie complète de l'œuvre de Francesco Orlando 1960-2014
}

\section{Luciano Pellegrini}

\section{Q OpenEdition \\ 1 Journals}

Édition électronique

URL : http://journals.openedition.org/rief/661

DOI : 10.4000/rief.661

ISSN : 2240-7456

Éditeur

Seminario di filologia francese

Référence électronique

Luciano Pellegrini, «Bibliographie complète de l'œuvre de Francesco Orlando 1960-2014 », Revue italienne d'études françaises [En ligne], 4 | 2014, mis en ligne le 15 décembre 2014, consulté le 15 septembre 2020. URL : http://journals.openedition.org/rief/661

Ce document a été généré automatiquement le 15 septembre 2020.

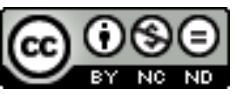

Les contenus de la RIEF sont mis à disposition selon les termes de la Licence Creative Commons Attribution - Pas d'Utilisation Commerciale - Pas de Modification 4.0 International. 


\title{
Bibliographie complète de l'œuvre de Francesco Orlando 1960-2014
}

\author{
Luciano Pellegrini
}

\section{NOTE DE L'ÉDITEUR}

Francesco Orlando (1934 - 2010), élève de Tomasi di Lampedusa, a été un des plus réputés spécialistes italiens de la littérature française et surtout un théoricien de la littérature dont les travaux, traduits en français et en anglais, se sont signalés pour leur originalité et leur rigueur. Il est en particulier l'auteur d'un cycle de quatre volumes (Einaudi) où il présente sa théorie freudienne de la littérature; d'un essai où il étudie les objets désuets dans l'imagination littéraire (Einaudi, Yale University Press et Classiques Garnier) ; d'une interprétation du Guépard de Lampedusa (Einaudi et Garnier) ; du roman La doppia seduzione (Einaudi).

Cette bibliographie contient le répertoire complet des mononographies, articles, préfaces, mémoires et ouvrages de fiction de Francesco Orlando, publiés pendant sa vie et posthumes, ainsi qu'une sélection d'entretiens et articles de presse. Pour un inventaire exhaustif des interventions journalistiques et des entretiens écrits, audio ou vidéo nous renvoyons à la bibliographie contenue dans Sei lezioni per Francesco Orlando. Teoria ed ermeneutica della letteratura (textes recueillis par P. Amalfitano et A. Gargano, Pisa, Pacini, «I Libri dell'Associazione Malatesta », 2014, p. 291-325) et dans la section consacrée à l'auteur sur le site internet www.mimesis.education/

\section{Monographies}

1. L'opera di Louis Ramond, Milano, Feltrinelli, 1960. [Traduction : France 2011]

2. Rotrou dalla tragicommedia alla tragedia, Torino, La Bottega d'Erasmo, 1963.

3. Infanzia, memoria e storia da Rousseau ai romantici, Padova, Liviana, 1966.

4. Lettura freudiana della "Phèdre», Torino, Einaudi, 1970. [Repris dans Due letture freudiane (1990). Traductions : États-Unis 1978 et France 1986] 
5. Per una teoria freudiana della letteratura, Torino, Einaudi, 1973. [Traductions : États-Unis 1978 et Pologne 1987]

6. Lettura freudiana del «Misanthrope »-e due scritti teorici, Torino, Einaudi, 1979. [Repris dans Due letture freudiane (1990)]

7. Illuminismo e retorica freudiana, Torino, Einaudi, 1982.

8. Le costanti e le varianti. Studi di letteratura francese e di teatro musicale, Bologna, Il Mulino, 1983.

9. Per una teoria freudiana della letteratura, nouvelle édition augmentée, Torino, Einaudi, 1987.

10. Due letture freudiane: Fedra e il Misantropo, Torino, Einaudi, 1990.

11. Per una teoria freudiana della letteratura, nouvelle édition augmentée, Torino, Einaudi, 1992.

12. Gli oggetti desueti nelle immagini della letteratura. Rovine, reliquie, rarità, robaccia, luoghi inabitati e tesori nascosti, Torino, Einaudi, 1993. [Nouvelles éditions : Torino 1994 et 2015. Traductions : États-Unis 2006 et France 2010]

13. L'altro che è in noi. Arte e nazionalità (Lezione Sapegno 1996), Torino, Bollati Boringhieri, 1996. [Traduction : en anglais (Italie) 1999)]

14. Illuminismo, barocco e retorica freudiana, Torino, Einaudi, 1997.

15. L'intimità e la storia. Lettura del «Gattopardo», Torino, Einaudi, 1998. [Traduction: France 2014]

16. Per una teoria freudiana della letteratura, nouvelle édition, Torino, Einaudi, 2000.

17. S. Timpanaro, F. O., Carteggio su Freud (1971-1977), Pisa, Scuola Normale Superiore, 2001.

18. Infanzia, memoria e storia da Rousseau ai romantici, avec une postface de S. Zatti, Pisa, Pacini, 2007.

\section{Postuma}

1. L'artificio contro la natura nel mondo di Baudelaire, éd. et postface de L. Pellegrini, Chieti, Solfanelli, « Arethusa », 2014.

2. Gli oggetti desueti nelle immagini della letteratura. Rovine, reliquie, rarità, robaccia, luoghi inabitati e tesori nascosti, nouvelle édition augmentée, Torino, Einaudi, à paraître.

\section{Directions d'ouvrages collectifs, éditions}

1. Giuseppe Tomasi di Lampedusa. Cento anni dalla nascita, quaranta dal «Gattopardo", Atti del Convegno, Palermo 12-14 dicembre 1996, Palermo, Edizioni Assessorato alla Cultura, 1999.

2. C. Samonà, Fratelli e tutta l'opera narrativa, Milano, Mondadori, 2001.

\section{Articles et préfaces en italien}

1. «Il sogno di Erode e i motivi della Mariane ", dans Saggi e ricerche di letteratura francese II, Milano, Feltrinelli, 1961, p. 31-79. [Repris dans Le costanti e le varianti (1983)]

2. "Il tema della méprise nelle tre novelle parigine di Mérimée ", dans Saggi e ricerche in memoria di Ettore Li Gotti, vol. II, Palermo, Mori \& Figli, 1962, p. 392-416. [Repris dans Le costanti e le varianti (1983)]

3. «La morte falsa e vera nel teatro di Rotrou », dans Studi francesi, 16, janvier-avril 1962, p. 31-50.

4. «Un motivo barocco e la struttura della Rodogune », dans Saggi e ricerche di letteratura francese III, Milano, Feltrinelli, 1963, p. 37-65. [Repris dans Le costanti e le varianti (1983)] 
5. «Su tre versi dell'Andromaque », dans Saggi e ricerche di letteratura francese, IV, Torino, La Bottega d'Erasmo, 1963, p. 62-81. [Repris dans Le costanti e le varianti (1983)]

6. «Mallarmé e la fede perduta (lettura di Sainte)", dans Rivista di Letterature Moderne e Comparate, 2, juin 1964, p. 85-97. [Repris dans Le costanti e le varianti (1983)]

7. « Rousseau e la nascita di una tradizione letteraria : il ricordo d'infanzia, I », dans Belfagor, vol. XX, 1, janvier 1965, p. 11-33.

8. «Rousseau e la nascita di una tradizione letteraria : il ricordo d'infanzia, II », dans Belfagor, vol. XX, 3, mai 1965, p. 411-433.

9. «Su Chateaubriand e Stendhal memorialisti : ricordi d'infanzia e rivoluzione », dans Annali della Scuola Normale Superiore di Pisa, série II, vol. XXXIV, fasc. I-II, Pisa 1965, p. 51-96.

10. « I ricordi d'infanzia di due memorialisti romantici. I. Il prezzo dei ricordi (Lamartine). II. La figlia della mésalliance (G. Sand) », Pisa, Industrie Grafiche V. Lischi \& Figli, 1965, p. 1-42.

11. «Charles Nodier memorialista : una infanzia sotto la rivoluzione », dans Critica storica, 3, mai 1965, p. 279-98.

12. «Baudelaire e la sera (lettura di Harmonie du soir)», dans Paragone, 196, juin 1966, p. 44-73. [Repris dans Le costanti e le varianti (1983). Traduction : France 2009]

13. « Ancora sull'esegesi critica di Sainte », dans Studi francesi, 28, janvier-avril 1966, p. 87-88.

14. «Baudelaire », dans I Protagonisti della Storia Universale, 114, Milano, Compagnia Edizioni Internazionali, 1967. [Repris dans Le costanti e le varianti (1983). Traduction : Argentine 1980]

15. «Il recente e l'antico nel cap. I, 18 di Le Rouge et le Noir », dans Belfagor, anno XXII, fasc. VI, novembre 1967, p. 661-80. [Repris dans Le costanti e le varianti (1983)]

16. «La Nuit et l'aube dans le IV acte du Venceslas de Rotrou», dans Cahiers de l'Association Internationale des études Françaises, 20, mai 1968, p. 137-147. [Repris dans Le costanti e le varianti (1983)]

17. «Le due facce dei simboli in un poema in prosa di Mallarmé ", dans Strumenti critici, 7, octobre 1968, p. 378-412. [Repris dans G. P. Caprettini, D. Corno (dir.), Letteratura e semiologia in Italia, Torino, Rosenberg \& Sellier, 1979, p. 139-179; ainsi que dans Le costanti e le varianti (1983)]

18. «Proust, Sainte-Beuve e la ricerca in direzione sbagliata », dans Critica e storia letteraria. Studi offerti a Mario Fubini, vol. I, Padova, Liviana, 1970, p. 226-50. [Repris dans M. Proust, Contre Sainte-Beuve, Torino, Einaudi, 1974, p. VII-XXXVII.]

19. «Marcel Proust dilettante mondano, e la sua opera », dans Nuovi Argomenti, 25, janvierfévrier 1972, p. 83-98. [Repris dans Proustiana, Atti del Convegno Internazionale di Studi sull'opera di Marcel Proust, organisé par la Società Universitaria per gli Studi di Lingua e Letteratura Francese (Venezia, 11 dicembre 1971), Padova, Liviana, 1973, p. 167-184 ; ainsi que dans La fondazione della Cgil (1906) [annexe de Il Manifesto], éd. E. Bouchard, R. Gagliardi et G. Polo, Roma, Fratelli Spada, 1993, p. 51-63, et dans Le parole e le cose (2013)]

20. «Proposte per una semantica del Leit-Motiv nell'Anello del Nibelungo », dans Nuova Rivista Musicale Italiana, 2, avril-juin 1975, p. 230-247. [Repris dans Le costanti e le varianti (1983)]

21. « Saggio introduttivo », dans S. Freud, Il motto di spirito e la sua relazione con l'inconscio, Torino, Boringhieri, 1975, p. 15-29.

22. «Lettura freudiana del Misanthrope », dans Micromégas, 2-3, 1976, p. 19-95.

23. «Su teoria della letteratura e divisione del lavoro intellettuale », dans Strumenti critici, 29, février 1976, p. 105-125. [Repris dans Lettura freudiana del «Misanthrope » (1979) et dans Per una teoria freudiana della letteratura (1987)]

24. «Preambolo a una lettura freudiana del Misanthrope », dans Strumenti critici, 31, octobre 1976, p. 384-403. 
25. «Baudelaire e il "valore dormente". (Lettura di Le Guignon)», dans Nuovi argomenti, 59-60, juillet-décembre 1978, p. 41-52. [Repris dans Le costanti e le varianti (1983). Traduction: France 2014]

26. « Retorica dell'illuminismo e negazione freudiana », dans A. Gargani (dir.), Crisi della ragione, Torino, Einaudi, 1979, p. 127-146. [Traduction : France 1980]

27. « Logica falsa e prestigio vano : una lettera di M. de Charlus » dans G. Giorgi, A. Principato, E. Biancardi, M. C. Bertoletti (dir.), Studi di cultura francese ed europea in onore di Lorenza Maranini, Fasano, Schena, 1983, p. 463-472. [Traduction : France 2010]

28. «L'ultima festa dell'Ancien Régime. Mozart e le commedie francesi », dans Scritti in onore di Giovanni Macchia, vol. II : Le dimensioni dello spettacolo, Mondadori, Milano 1983, p. 426-441. [Repris dans Le costanti e le varianti (1983)]

29. « Mito e storia ne L'Anello del Nibelungo », dans G. Bevilacqua (dir.), Parole e musica : l'esperienza wagneriana nella cultura fra Romanticismo e Decadentismo, Atti del convegno internazionale di studio per le celebrazioni del centenario wagneriano (Venezia 21-23 febbraio 1983), Firenze, Olschki, 1983, p. 53-70. [Repris dans Intersezioni, 2, août 1983, p. 347-360. Traduction : France 1987]

30. "Vathek o la dannazione dell'enfant gâté », dans Saggi e ricerche di letteratura francese XXIII, Roma, Bulzoni, 1984, p. 277-303. [Repris dans M. Matucci (dir.), Lumières et illuminisme, Actes du Colloque international (Cortona, 3-6 octobre 1983), Pisa, Pacini, 1985, p. 187-204, ainsi que dans Beckford, Vathek. Racconto arabo, Venezia, Marsilio, 1996, p. 9-36. Traduction : française (Italie) 2011]

31. «Letteratura e psicanalisi : alla ricerca dei modelli freudiani », dans A. Asor Rosa (dir.), Letteratura italiana. Volume IV. L'interpretazione, Torino, Einaudi, 1985, p. 549-587. [Repris dans Per una teoria freudiana della letteratura (1987). Version américaine : États-Unis 1984]

32. «"Sapere" contro "vedere" ", dans Alfabeta, 72, mai 1985, p. 15.

33. «Spunti introduttivi per la lettura di La Recherche di Proust, dans M. Fantuzzi (dir.), Il romanzo. Origine e sviluppo delle strutture narrative nella letteratura occidentale, Associazione di cultura classica. Delegazione di Pontedera, Liceo scientifico XXV aprile, Pisa, ETS, 1987, p. 233-241.

34. « La fine della preistoria nella musica del Ring : figlie del Reno, valchirie, norne », dans Nuova Rivista Musicale Italiana, 4, octobre-décembre 1988, p. 663-679. [Traduction : Allemagne 1991]

35. «Verso un contributo al codice dei "topoi” letterari moderni : presentazione di una ricerca » , dans R. Antonelli, F. Beggiato, A. Ferrari, A. Solimena (dir.), Miscellanea di studi in onore di Aurelio Roncaglia, a cinquant'anni dalla sua laurea, vol. III, Modena, Mucchi, 1989, p. 933-944.

36. «Dodici regole per la costruzione d'un paradigma testuale », dans L'Asino d'oro, 1, 1990, p. 122-135. [Repris dans Per una teoria freudiana della letteratura (1992)]

37. «Parole dette ai funerali di Carmelo Samonà », dans Belfagor, anno XLV, fasc. III, 1990, p. 307-310.

38. « Una lettera di Orlando sull'intervista a Timpanaro », dans Allegoria, 9, 1991, p. 91-92.

39. " "Topoi" del realismo. La metamorfosi dei colori », dans Realismo ed effetti di realtà nel romanzo dell'Ottocento, Roma, Bulzoni, «I Libri dell'Associazione Sigismondo Malatesta », 1993, p. 3-10. [Traduction : États-Unis 1993]

40. «Per una ricerca di costanti nel teatro europeo : il dato di fatto falso », dans L. Innocenti, F. Marucci, P. Pugliatti (dir.), Semeia. Itinerari per Marcello Pagnini, Bologna, Il Mulino, 1994, p. 79-87.

41. «Il dato di fatto falso. Tecnica teatrale e visione del mondo", dans S. Carandini (dir.), Il valore del falso. Errori, inganni, equivoci sulle scene europee in epoca barocca, Roma, Bulzoni, «I Libri dell'Associazione Sigismondo Malatesta », 1994, p. 27-37. 
42. Fantasia siciliana, razionalismo inglese, dans R. Andò (dir.), Ricordo di Antonio Pasqualino, Palermo, Museo Internazionale delle Marionette Antonio Pasqualino. Associazione per la conservazione delle tradizioni popolari, [s. d.], p. 17-23.

43. «Costanti tematiche, varianti estetiche e precedenti storici », préface à M. Praz, La carne, la morte e il diavolo nella letteratura romantica, Firenze, Sansoni, 1996, p. VII-XXI.

44. «Trama del racconto e trama dei motivi nell'Oro del Reno", dans R. Wagner, Das Rheingold, Milano, Edizioni del Teatro alla Scala, 1996, p.104-115. [Repris sous le titre «Natura e antinatura, preistoria e storia», dans R. Wagner, Das Rheingold, Fondazione Teatro del Maggio Musicale Fiorentino, Bologna, Pendragon, 2007, p. 20-37.]

45. «Buio e sole di un'ambigua foresta », dans R. Wagner, Siegfried, Milano, Edizioni del Teatro alla Scala, 1998, p. 119-29. [Repris dans R. Wagner, Siegfried, Fondazione Teatro del Maggio Musicale Fiorentino, Firenze, Giunti, 2008, p. 210-225.]

46. «Un elmo magico fra mito e modernità », dans R. Wagner, Götterdämmerung, Milano, Edizioni del Teatro alla Scala, 1998, p. 182-193. [Repris dans R. Wagner, Götterdämmerung, Fondazione Teatro del Maggio Musicale Fiorentino, Firenze, Giunti, 2009, p. 196-213]

47. « Il rapporto uomo-opera e la questione del giudizio di valore. Lettera aperta a Romano Luperini », dans Allegoria, 32, mai-août 1999, p. 134-137.

48. « Statuti del soprannaturale nella narrativa», dans F. Moretti (dir.), Il romanzo, I, La cultura del romanzo, Torino, Einaudi, 2001, p. 195-226.

49. «Teoria della letteratura, letteratura occidentale, alterità e particolarismi », dans U. M. Olivieri (dir.), Un canone per il terzo millennio. Testi e problemi per lo studio del Novecento tra teoria della letteratura, antropologia e storia, Milano, Bruno Mondadori, 2001, p. 63-87.

50. «Suoni flebili e opachi », dans C. Samonà, Fratelli e tutta l'opera narrativa, éd. F. O., Milano, Mondadori, 2001, p. V-XVII.

51. «Prefazione" à E. Zinato, Il vero in maschera. Dialogismi galileiani : idee e forme nelle prose scientifiche del Seicento, Napoli, Liguori, 2003, p. 1-4.

52. «Voltaire : prima, durante, dopo », préface à Voltaire, Racconti, facezie, libelli, éd. G. Iotti, Torino, Einaudi, « Biblioteca della Pléiade », 2004, p. XIII-XXX.

53. «Per un libro mai scritto », dans G. Papoff, G. Rocca (dir.), Stéphane Mallarmé. Un secolo di poesia, Atti del convegno internazionale, Napoli 11-12 novembre 1999, Napoli, La Città del Sole, 2004, p. 27-50.

54. «Festa corale e pene personali : una costante operistica », dans M. Fusillo, M. Polacco (dir..), La letteratura e le altre arti, Atti del Convegno dell'Associazione di Teoria e Studi di Letteratura Comparata, L'Aquila, febbraio 2004, dans Contemporanea, vol. 3, Pisa-Roma 2005, p. 33-39.

55. «Pelléas, Wagner, musica nazionale, "immagini" sonore», dans C. Debussy, Pelléas et Mélisande, Milano, Edizioni del Teatro alla Scala, 2005, p. 65-83.

56. «Qualche considerazione sulle funzioni letterarie del comico », dans E. Marinai, S. Poeta, I. Vazzaz (dir.), Comicità negli anni Settanta. Percorsi eccentrici di una metamorfosi fra teatro e media, Pisa, ETS, 2005, p. 35-43.

57. « Prefazione alla ristampa » de Infanzia, memoria e storia da Rousseau ai romantici, Pisa, Pacini, 2007, p. 5-14.

58. « 150 anni dalla nascita di Freud. La psicanalisi nella cultura del nostro tempo », dans Rivista di Psicanalisi, 1, janvier-mars 2007, p. 209-17.

59. «Le unità di un testo letterario e le classi di Matte Blanco », dans A. Ginzburg, R. Lombardi (dir.), L'emozione come esperienza infinita. Matte Blanco e la psicanalisi contemporanea, Milano, FrancoAngeli, 2007, p. 213-226. [Repris sous le titre « Reminiscenze letterarie e "classi" : una autoanalisi ", dans S. Teroni (dir.), La voce della poesia, Firenze, Nicomp, 2009, p. 17-40] 
60. «Tenerezza familiare e separazione tragica», dans R. Wagner, Die Walküre, Fondazione Teatro del Maggio Musicale Fiorentino, Bologna, Pendragon, 2007, p. 18-39.

61. «A che cosa serve la letteratura?» dans P. Tamassia (dir.), A che serve la letteratura? (e il suo insegnamento), Lisi, Taranto 2007, p. 59-62. [Voir aussi sur le site <www.filologiafrancese.it>. Repris dans D. Ragone (dir.), Per Francesco Orlando. Testimonianze e ricordi, Pisa, ETS, 2012]

62. «Fra la persona e il testo : contesti, allusioni, reticenze, trasfigurazioni », dans C. De Carolis (dir.), La biografia, Roma, Bulzoni, «I Libri dell'Associazione Sigismondo Malatesta », 2008, p. 225-247.

63. «Se fuggi inseguo, se insegui fuggo », dans G. Bizet, Carmen, Fondazione Teatro del Maggio Musicale Fiorentino, Bologna, Pendragon, 2008, p. 194-211. [Repris dans S. Sebastiani (éd.), Donne in rivolta. Tra arte e memoria, Istituto Italiano di Scienze Umane, Bologna, Il Mulino, 2011, p. 187-205]

64. «Baudelaire et le soir", trad. de A. et P.-A. Claudel, dans L'Année Baudelaire, 11/12, 2007-2008, p. 147-180.

65. «Codici letterari e referenti di realtà in Auerbach», dans R. Castellana (dir.), La rappresentazione della realtà. Studi su Erich Auerbach, Atti del Convegno della Scuola di dottorato dell'Università di Siena, L'interpretazione, Siena, 29-30 aprile 2008, Siena, Artemide, 2009, p. 17-62. [Voir aussi : <http://www.mimesis.education/>. Version française : 2009]

66. «Tra il fiabesco e il coniugale. Riflessioni attorno a La donna senz'ombra », dans R. Strauss, Die Frau ohne Schatten, Fondazione Teatro del Maggio Musicale Fiorentino, Firenze, Giunti, 2010, p. 176-195.

\section{Postuma}

1. «Prefazione» à M. Proust, Corrispondenza con la madre (1887-1905), éd. M. Patti, Lanciano, Carabba, 2010.

2. «Premessa » à F. Accorsi, C. Tognarelli (dir.), Testo e commento. Seconda giornata di studi della Scuola di Dottorato in Letterature e Filologie moderne [Università di Pisa], Pisa, Felici, 2011, p. 15-18.

3. «Opera letteraria e autore. Storia e teoria di un rapporto », dans Lezioni Urbinati 2003-2009, éd. D. Tagliafico, Torino, Codice Edizioni, 2011, p. 53-70.

4. «Sui limiti del biografismo e dell'ideologismo nella critica letteraria. Due inediti », éd. S. Brugnolo, préface de E. Zinato, dans I Nuovi Samizdat, 56, Padova 2011.

5. Marcel Proust dilettante mondano, e la sua opera, dans Le parole e le cose, 14 novembre 2013, <http://www.leparoleelecose.it/?p=12826\#more-12826>

6. « Il patto col lettore (Rousseau) », dans J.-J. Rousseau, Rousseau giudice di Jean-Jacques, Grumo Nevano (Na), Marchese 2014, p. 423-456.

\section{Articles traduits ou publiés en langue non italienne}

1. «La découverte du souvenir d'enfance au premier livre des Confessions », dans Annales de la Société Jean-Jacques Rousseau, 37 (1966-1968), 1968, p. 149-173.

2. «Propositions pour une sémantique du leit-motiv dans L'Anneau du Nibelung », dans Musique en jeu, 17, janvier 1975, p. 73-86. [it. : 1983]

3. «Rhétorique des Lumières et dénégation freudienne", dans Poétique, 41, février 1980, p. 78-89.

4. «D’un triangle: Freud, Paris, les études littéraires en Italie», dans Micromégas, 3, 1980, p. 63-69. 
5. «Freud and Literature. Eleven Ways he did it », dans Poetics, 13, 1984, p. 361-380. [it. : 1985]

6. «Le Mythe et l'histoire dans le Ring de Wagner", dans Ph. Berthier, K. Ringger (dir.), Littérature et Opéra, Grenoble, Presses universitaires de Grenoble, 1987, p. 99-108. [it. : 1983]

7. «Literatura Pomį̨, zadmiarem a Niedostatkiem retoryki » [La letteratura fra eccesso $e$ difetto di retorica, chap. IV de Per una teoria freudiana della litteratura (1987)], trad. de J. Ugniewska, dans PamiĘtnik Literacki, LXXVIII, 3, 1987, p. 261-273. [it. : 1973]

8. « Powrót Stłumienia W Serii Treści » [Il ritorno del represso nella serie dei contenuti, chap. V de Per una teoria freudiana della letteratura (1987)], trad. de J. Ugniewska, dans PamiĘtnik Literacki, LXXVIII, 3, 1987, p. 275-286. [it. : 1973]

9. «Das Ende der Vorgschichte in der Musik der Tetralogie: Rheintöchter, Walküren und Nornen », dans W. Storch (dir.), Der Ring am Rhein, vol. I, Berlin, Hentrich, 1991, p. 75-85. [it. : 1988]

10. "Topoi » of Realism: The Metamorphosis of Colors, trad. de J. Clegg, dans W. Sollors (dir.), The Return of Thematic Criticism, Cambridge, Harvard University Press, 1993, p. 209-226. [it. : 1993]

11. «The Native and the Outsider: Literature and Nationality », trad. de A. Thompson, dans G. Sertoli, G. Miglietta (dir.), Transiti letterari e culturali, Atti del XVIII Congresso nazionale dell'A.I.A., Genova 30 settembre-2 ottobre 1996, Trieste, E.U.T., 1999, p. 1-15. [it. : 1996]

12. «Bilan du Congrès », dans L. Omacini (dir.), Le Statut du sujet dans le récit de mémoire, Actes du Colloque de Venise 28-29 novembre 1997, Padova, Unipress, 1999, p. 149-158.

13. «Temps de l'histoire, espace des images", dans J.-Cl. Berchet, Ph. Berthier (dir.), Chateaubriand mémorialiste. Colloque du cent-cinquantenaire (1848-1998), Actes du colloque tenu à l'Université Sorbonne-Nouvelle (Paris III), 4-6 juin 1998, Genève, Droz, 2000, p. 109-118.

14. " "Savoir" contre "voir". Métamorphose et métaphore », dans M. Carbone, E. Sparvoli (dir.), Proust et la philosophie aujourd'hui, Actes du colloque de Gargnano, 28-30 septembre 2006, Pisa, ETS, 2008, p. 19-32.

15. «Codes littéraires et référents chez Auerbach», dans P. Tortonese (dir.), Erich Auerbach. La Littérature en perspective, Paris, Presses de la Sorbonne Nouvelle, 2009, p. 211-264. [it. : 2009]

16. «Logique fausse et vain prestige : une lettre de Monsieur de Charlus », dans M. Bertini, A. Compagnon, Morales de Proust, Cahiers de littérature française, IX-X, Bergamo-Paris, SestanteL'Harmattan, 2010, p. 15-26. [it. : 1983]

17. «L'Histoire des montagnes et l'harmonie du monde chez Ramond de Carbonnières ", trad. de É. Faure, dans G. Bertrand, A. Guyot (dir.), Des «passeurs» entre science, histoire et littérature, Grenoble, ELLUG, Université Stendhal, p. 167-220. [Traduction du chap. IV de L' opera di Louis Ramond (1960)]

18. "Vathek ou la damnation de l'enfant gâté », dans Revue italienne d'études françaises, 1, 2011, p. 275-301, <http://www.rief.it>. [it. : 1984]

19. «Baudelaire et la "valeur dormante". Sur Le Guignon », trad. de S. Migliarina, dans L'Année Baudelaire, 17, 2013, p. 15-26. [it. : 1978]

\section{Comptes rendus ${ }^{1}$}

1. J.-J. Seylaz, Les "Liaisons dangereuses" et la création romanesque chez Laclos (Genève-Paris, Droz- Minard, 1958), dans Studi Francesi, 7, janvier-avril 1959, p. 103-105.

2. R. Pomeau, La Religion de Voltaire (Paris, Nizet, 1956), dans Rivista di Letterature Moderne e Comparate, vol. XII, 1, mars 1959, p. 83-86.

3. C. Cherpack, The Call of Blood in French Classical Tragedy (Baltimore, Hopkins, 1958), dans Rivista di Letterature Moderne e Comparate, vol. XII, 2, juin 1959, p. 170-72. 
4. J. Starobinski, Jean-Jacques Rousseau. La Transparence et l'Obstacle (Paris, Plon, 1957), dans Rivista di Letterature Moderne e Comparate, vol. XIII, 1-2, juin 1960, p. 116-120.

5. «L'Edipo cristiano e quello pagano », compte rendu de G. Paduano, Lunga storia di Edipo Re. Freud, Sofocle e il teatro occidentale (Torino, Einaudi, 1994), dans La Rivista dei Libri, 10, octobre, p. 12-14. [Repris dans Illuminismo, barocco e retorica freudiana (1997)]

6. « Darwin, Freud, l'individuo e il caso », compte rendu de F. Moretti, Opere mondo. Saggio sulla forma epica dal «Faust» a "Cent'anni di solitudine» (Torino, Einaudi, 1994), dans La Rivista dei Libri, 2, février 1995, p. 19-21.

7. «Francesi, ancora uno sforzo!», compte rendu de A. Compagnon, Le Démon de la théorie. Littérature et sens commun (Paris, Seuil, 1998), dans La Rivista dei Libri, 3, mars 1999, p. 26-28.

\section{Articles de presse, entretiens}

1. «Francesco Orlando : psicanalisi e letteratura », réponses à un questionnaire de A. D’Andrea et D. Della Terza, dans Year book of Italian Studies, 1973-1975, p. 201-218.

2. « La fortuna di Wagner. A proposito del centenario dell'Anello del Nibelungo a Bayreuth » dans l'Unità, 2 octobre 1976, p. 3.

3. «Orlando : questa è una rivoluzione [à propos d'Ignacio Matte Blanco] », dans Panorama, 26 juillet 1982, p. 139.

4. « Logica "simmetrica" e storicità. Intervista su psicanalisi e letteratura », entretien accordé à F. Petroni, dans L' ombra d'Argo, 1-2, 1983, p. 143-151.

5. Arte, ideologia e forma in Todorov, entretien accordé à R. Ceserani, dans L'Indice, 4, mai 1985, p. 24-25.

6. Le reliquie e i tesori del testo, entretien accordé à M. Gulinucci et M. Serri, dans L'Espresso, 3 décembre 1988, p. 162.

7. I giovani e la trasmissione del sapere. Né lingue, né lettere per lo studente dimezzato, entretien accordé à G. Borgese, dans Corriere della Sera, 26 février 1988, p. 23.

8. Porte aperte per James Joyce [sur les Lezioni di letteratura inglese de Giuseppe Tomasi di Lampedusa], entretien accordé à L. Lilli, dans la Repubblica, 21 janvier 1990.

9. Date di nascita [sur Gli oggetti desueti], entretien accordé à F. Borrelli, dans Linea d'ombra, mai 1993, p. 69-72.

10. Malinconia storica [sur Chateaubriand], dans L'Indice, 11, novembre 1995, p. 18.

11. La letteratura e le cose. Conversazione tra Francesco Orlando e Claudio Pavone [1993], dans Parolechiave, 9, 1995, La memoria e le cose, Roma, Donzelli, 1995, p. 45-65.

12. Così era il Gattopardo, maestro generoso e crudele, entretien accordé à R. Minore, dans Il Messaggero, 18 août 1996, p. 16.

13. Un Gattopardo fra le dita, entretien accordé à G. Calcagno, dans La Stampa, 16 novembre 1996, p. 25.

14. Ma il « padre forte » è mai esistito ?, entretien accordé à F. Ghelli e A. Stara, dans Inchiesta, Padri e figli, 28, octobre-décembre 1998, p. 18-23.

15. Uomo siciliano, scrittore inglese, entretien accordé à R. Cirio, dans L'Espresso, 19 mars 1998, p. 105.

16. L'ultima zampata del Gattopardo, entretien accordé à M. Collura, dans Corriere della Sera, 25 avril 1998, p. 31.

17. Caro Asor Rosa perché uccidi il " Gattopardo »? - Replica a una stroncatura, dans la Repubblica, 24 septembre 1998, p. 36.

18. Il ritorno del « Gattopardo », dans la Repubblica, 25 octobre 1998, p. 12. 
19. Memoria privata e memoria collettiva. Una conversazione con Francesco Orlando, entretien accordé à G. Mazzoni, dans L'ospite ingrato. Annuario del Centro Studi Franco Fortini, Macerata, Quodlibet, 1999, p. 111-118.

20. Il mio Barthes fallito, entretien accordé à R. Andreotti, dans Alias, 6 mars 1999, p. 6.

21. Tomasi di Lampedusa. Liberamente ispirato [à propos du film Il manoscritto del principe de Roberto Andò], dans Corriere della sera, 10 avril 2000, p. 25.

22. L'eredità di Matte Blanco, entretien accordé à L. Sica, dans la Repubblica, 18 septembre 2004, p. 39.

23. «Una riflessione sulla narrativa di Cerami », dans Athenet, 18, décembre 2006, p. 7-9.

24. I realismi di Auerbach, entretien accordé à G. Tinè, dans Allegoria, 56, juillet-décembre 2007, p. 36-51.

25. Conversazione con Francesco Orlando, propos recueillis par A. Diazzi et F. Pianzola, dans Enthymema, I, 2009, p. 187-215, <http://riviste.unimi.it/index.php/enthymema>

26. A colloquio con Francesco Orlando, entretien accordé à L. Della Pietà, dans Letteratura e Letterature, 3, 2009, p. 163-176.

27. Intervista a Francesco Orlando su «La doppia seduzione», entretien accordé à E. Zinato, <http:// www.einaudi.it/speciali/Francesco-Orlando-La-doppia-seduzione-intervista-EmanueleZinato>.

28. Letteratura e omosessualità, entretien accordé à F. Marcoaldi, dans la Repubblica, 2 mars 2010, p. 55.

\section{Postuma}

1. Forse per essere più ascoltati in Italia la via più diretta non passa per il Tevere o l'Arno, ma per la Senna o l'Hudson, entretien accordé à D. Ragone, dans Sant'Anna News, 35, septembre 2010, p. 1 et 6-10. [Repris dans D. Ragone (dir.), Per Francesco Orlando. Testimonianze e ricordi, Pisa, ETS, 2012].

2. Conversazioni con Francesco Orlando, propos recueillis par V. Baldi, dans Allegoria, 65-66, janvier-juin 2012.

\section{Mémoires et correspondances}

1. Ricordo di Lampedusa, Milano, All'insegna del Pesce d'oro, 1963.

2. Ricordo di Lampedusa (1962) seguito da Da distanze diverse (1996), Torino, Bollati Boringhieri, 1996. [Traductions : France 1996 et Espagne 2006].

3. Timpanaro, F. O., Carteggio su Freud (1971-1977), Pisa, Scuola Normale Superiore, 2001.

\section{Ouvrages de fiction}

1. La doppia seduzione, roman, Torino, Einaudi, 2010.

\section{Ouvrages traduits}

1. Toward a Freudian Theory of Literature. With an analysis of Racine's "Phèdre", trad. de Charmaine Lee, Baltimore and London, The Johns Hopkins University Press, 1978. [it. : 1970 et 1973]

2. El mundo de Charles Baudelaire, Buenos Aires, Centro editor de America latina, 1980. [it. : 1967] 
3. Lecture freudienne de "Phèdre", trad. par D. et Th. Aron, Paris, Les Belles Lettres, "Annales littéraires de l’Université de Besançon », 1986. [it. : 1970]

4. Un souvenir de Lampedusa, suivi de à distances multiples, trad. de M. Balzamo, Paris, L'Inventaire, 1996. [it. : 1996]

5. Recuerdo de Lampedusa, seguido de Con otra distancia, trad. de J. A. Méndez Borra, Valencia, PreTextos, 2006. [it. : 1996]

6. Obsolete Objects in the Literary Imaginatio Ruins, Relics, Rarities, Rubbish, Uninhabited Places, and Hidden Treasures, trad. de G. Pihas et D. Seidel, avec la collaboration de A. Grego, New Haven \& London, Yale University Press, 2006. [it. : 1994]

7. Les Objets désuets dans l'imagination littéraire. Ruines, reliques, raretés, rebuts, lieux inhabités et trésors cachés, trad. de Paul-André e Aurélie Claudel, Paris, Classiques Garnier, 2010. [it. 1994. Deuxième édition avec une préface de Carlo Ginzburg : Paris 2013]

8. L'Intimité et l'Histoire. Lecture du Guépard, trad. de C. De Carolis, Paris, Classiques Garnier, 2014. [it. : 1998]

\section{NOTES}

1. Pour le répertoire des nombreuses notes de lectures rédigées entre 1957 et 1962 pour la section XVIII ${ }^{e}$ siècle de la "Rassegna bibliografica » de Studi francesi, je renvoie à Sei lezioni per Francesco Orlando (cit.) et au site internet www.mimesis.education/.

INDEX

Mots-clés : Orlando (Francesco) 